
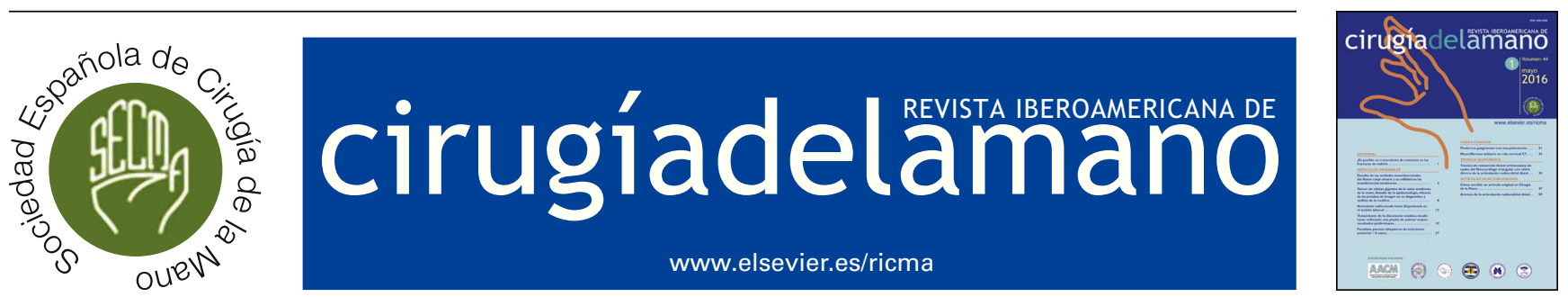

ARTÍCULO DE ACTUALIZACIÓN

\title{
Inestabilidad lunopiramidal
}

\author{
M. Ocampos Hernández ${ }^{a, b, *}$, F. Corella Montoya ${ }^{a, b}, M$. del Cerro Gutiérrez ${ }^{b}$ \\ y B. del Campo Cereceda ${ }^{a}$
}

\author{
a Hospital Universitario Infanta Leonor, Madrid, España \\ b Unidad de Cirugía de Mano, Hospital Beata María Ana, Madrid, España
}

Recibido el 8 de septiembre de 2016; aceptado el 14 de septiembre de 2016

Disponible en Internet el 19 de octubre de 2016

\author{
PALABRAS CLAVE \\ Artroscopia de \\ muñeca; \\ Ligamento \\ lunopiramidal; \\ Volar Intercalated \\ Segmentary \\ Instability (VISI); \\ Inestabilidad \\ carpiana
}

\section{KEYWORDS}

Wrist arthroscopy;

Lunotriquetral

ligament;
Resumen Históricamente, la inestabilidad en el lado radial de la muñeca ha recibido mucha más atención que la inestabilidad en el lado ulnar, a pesar de ser una patología relativamente frecuente. Como consecuencia de ello, suele darse un retraso en el diagnóstico correcto y el tratamiento apropiado. Las lesiones del ligamento lunopiramidal pueden ser parciales o completas, y estas últimas pueden estar asociadas a lesión de los estabilizadores secundarios. Cuando se lesionan estos estabilizadores es cuando se produce una inestabilidad estática del carpo conocida como Volar Intercalated Segmentary Instability (VISI). Entre los test clínicos más utilizados para detectar una inestabilidad del ligamento lunopiramidal están el Ballottement test, descrito por Reagan, y el Shear test, descrito por Kleinman, aunque ambos poseen una sensibilidad y una especificidad bajas. Para el diagnóstico de estas lesiones se emplean técnicas como la radiografía simple (que suele ser normal si no hay una inestabilidad estática del carpo), la artrografía y la RMN, entre otras, aunque el gold standard lo constituye la artroscopia de muñeca. No existe un consenso en cuanto al tipo de tratamiento óptimo, ya que la mayoría de los estudios publicados son retrospectivos de nivel iv sin medidas objetivas estandarizadas mediante instrumentos validados. Además, en su mayoría describen los resultados del tratamiento tardío, ya que no se suelen diagnosticar en la fase aguda. Dentro de los tratamientos descritos se encuentran el tratamiento artroscópico (desbridamiento/termocoagulación asociados o no a estabilización con agujas de Kirscher), la reparación abierta, la reconstrucción del ligamento y la artrodesis del ligamento lunopiramidal.

( 2016 SECMA. Publicado por Elsevier España, S.L.U. Este es un artículo Open Access bajo la licencia CC BY-NC-ND (http://creativecommons.org/licenses/by-nc-nd/4.0/).

\section{Lunotriquetral instability}

Abstract Radial-sided wrist instability has historically received greater attention than ulnarsided instability despite being a relatively frequent pathology. As a result, there is often a delay in correct diagnosis and appropriate treatment. Lunotriquetral ligament injuries can be

\footnotetext{
* Autor para correspondencia.

Correo electrónico: Montse.ocampos@gmail.com (M. Ocampos Hernández).
} 
Volar Intercalated Segmentary Instability (VISI); Carpal instability partial or complete, and the latter may be associated with injury of secondary stabilisers. When these stabilisers are injured a static carpal instability, known as Volar Intercalated Segmentary Instability (VISI), occurs. Among the most common clinical tests used to detect lunotriquetral instability are the ballottement test, described by Reagan, and the shear test, described by Kleinman, although both of them have a low sensitivity and specificity. Several techniques for the diagnosis of these injuries are used, such as X-rays (usually normal if there is no static instability of the carpus), arthrography and magnetic resonance imaging, although wrist arthroscopy is the reference standard for diagnosing these lesions. There is no consensus about which is the optimal treatment of lunotriquetral ligament tears, as the literature consists mostly of retrospective level iv studies without standardised objective measurements using validated instruments. Furthermore, most of them describe the results of late treatment due these lesions not usually being diagnosed in an acute phase. Among the treatments described are arthroscopic treatment (debridement/thermal shrinkage/pinning the lunotriquetral joint), open repair of the lunotriquetral ligament, soft tissue reconstruction, and lunotriquetral arthrodesis.

(c) 2016 SECMA. Published by Elsevier España, S.L.U. This is an open access article under the CC BY-NC-ND license (http://creativecommons.org/licenses/by-nc-nd/4.0/).
La integridad de los ligamentos lunopiramidal (LP) y escafolunar (EL) es esencial para el mantenimiento de una cinemática normal del carpo. La lesión del ligamento LP no es infrecuente, pero a menudo pasa desapercibida. En este artículo se revisa la inestabilidad LP en un contexto amplio desde su mecanismo de lesión hasta los tratamientos descritos.

\section{Anatomía y patomecánica}

El ligamento LP estabiliza la articulación LP. De forma similar a la estructura descrita en el ligamento EL, el ligamento LP tiene forma de $C$ y consta de 3 partes: dorsal, proximal o membranosa, y palmar. Las subregiones dorsal y palmar son verdaderos ligamentos compuestos de fibras de colágeno dispuestas de forma transversal, mientras que la porción membranosa sirve de puente entre ambas y está compuesta de fibrocartílago. La porción volar es la más gruesa y la más fuerte de las 3 y transmite la tendencia a la extensión del piramidal cuando se acopla a la superficie articular helicoidal del ganchoso. La subregión dorsal del ligamento es la más importante en restringir la rotación del semilunar ${ }^{1}$.

Al igual que sucede en las lesiones del ligamento EL, las lesiones del ligamento LP pueden ser parciales o completas sin lesión asociada de los estabilizadores secundarios (inestabilidad dinámica) o con lesión de estos (inestabilidad estática). Con la pérdida de integridad del ligamento LP, el piramidal tiende hacia la extensión mientras que el semilunar y el escafoides lo hacen hacia la flexión. Sin embargo, la lesión aislada del ligamento LP raramente es suficiente para permitir que el momento de flexión del escafoides flexione el semilunar. Es necesaria la lesión de los estabilizadores secundarios para producir una inestabilidad estática del carpo con flexión palmar semilunar o deformidad en Volar Intercalated Segment Instability (VISI). Probablemente jueguen un papel como restrictores secundarios tanto ligamentos dorsales como ligamentos volares en el carpo. Se ha demostrado que una lesión de los ligamentos radiocarpiano dorsal e intercarpiano dorsal asociada a una lesión del ligamento LP puede producir una deformidad en $\mathrm{VISI}^{2,3}$. Por otra parte, Trumble et al. ${ }^{4}$ han implicado al brazo ulnar del ligamento arcuato, cuya lesión, junto con el ligamento LP, también da lugar a una deformidad en VISI.

\section{Mecanismo de lesión}

El mecanismo de lesión del ligamento LP constituye un tema de debate y es menos conocido que el mecanismo de lesión del ligamento EL. Es posible que múltiples mecanismos puedan estar implicados como elementos causales ${ }^{5}$.

En el modelo descrito por Mayfield et al. ${ }^{6}$ se explica la progresión del ligamento EL hacia la lesión del ligamento LP e incluso una luxación perilunar, en que la lesión del ligamento LP queda encuadrada en el estadio III. En este modelo, el mecanismo es un traumatismo con la muñeca en posición de extensión, desviación ulnar y supinación mediocarpiana. Sin embargo, este modelo no justifica la lesión aislada del ligamento LP. Shin et al. ${ }^{7}$ han propuesto un modelo opuesto al descrito por Mayfield et al. ${ }^{6}$ para explicar las lesiones que comienzan en el lado ulnar y se extienden hacia el lado radial de la muñeca, cuyo mecanismo sería una caída con el antebrazo en supinación y la muñeca en extensión y desviación radial. Este modelo está secuenciado en 3 estadios:

- Estadio I: lesión del ligamento LP.

- Estadio II: lesión del ligamento LP más lesión de los ligamentos ulnocarpianos y de los ligamentos intercarpiano y radiocarpiano dorsales.

- Estadio III: estadio ॥ más lesión del ligamento EL y radioescafolunar con una potencial luxación perilunar dorsal.

Por último, en casos sin traumatismo previo la inestabilidad LP puede aparecer en el contexto de lesiones degenerativas 0 artritis inflamatoria ${ }^{7}$. 


\section{Diagnóstico}

\section{Examen clínico}

Las lesiones del ligamento LP producen dolor en el lado ulnar de la muñeca, que puede estar asociado a un «clunc» con la desviación ulnar de la misma y a una sensación de pérdida de fuerza. En lesiones completas con una inestabilidad estática del carpo la muñeca puede adoptar una deformidad en dorso de tenedor con una prominencia de la ulna distal.

Se han descrito varios test para detectar una inestabilidad LP:

Test de Reagan: la mano del paciente se mantiene en pronación fijando el semilunar con el pulgar y el índice de una mano del examinador mientras se desplaza el piramidal en sentido dorsal y volar con la otra mano. Un test positivo implica dolor, crepitación y mayor laxitud en este intervalo ${ }^{8}$.

Test de Kleinman: el antebrazo del paciente se mantiene en posición neutra y el codo en flexión. Se estabiliza el semilunar con una mano mientras se desplaza el pisiforme en sentido dorsal con la otra? .

Test de Derby: descrito por Christodoulou y Bainbridge ${ }^{10}$, está compuesto por 3 test clínicos:

- Primer test: se coloca la muñeca en dorsiflexión y desviación radial con el antebrazo en pronación y se ejerce una presión sobre el pisiforme con el pulgar desviando la muñeca hacia una posición en neutro. Con esta maniobra se observa un «clunc» al reducir el piramidal en su posición. El test es positivo si el paciente nota una mejoría en la fuerza de puño en esta posición.

- Segundo test: se coloca la muñeca en posición neutra y desviación radial con el antebrazo en pronación y se presiona hacia palmar la superficie dorsal del piramidal con el pulgar mientras se desvía la muñeca hacia radial. El test es positivo cuando reproduce el dolor.

- Tercer test: es el mismo gesto que el segundo test pero el pulgar del examinador ejerce una presión hacia dorsal sobre el pisiforme. El dolor disminuye con la desviación ulnar.

Test de la «tabaquera ulnar», delimitada entre el flexor ulnar del carpo y el extensor ulnar del carpo. Implica presión lateral sobre el piramidal mientras la muñeca se desvía hacia radial. Es positivo cuando reproduce los síntomas en el paciente ${ }^{11}$.

Todos estos test son sensibles pero poco específicos y pueden aparecer con otras lesiones en el lado ulnar de la muñeca.

\section{Examen radiológico}

Radiografía simple

Las radiografías estándar en las muñecas con lesiones del ligamento LP suelen ser normales en la mayoría de los pacientes. Cuando existe una disociación LP aparece una alteración en los arcos formados por la línea proximal y distal de la primera hilera del carpo y por la línea proximal de la segunda hilera del carpo (arcos de Gilula), lo que resulta en una traslación proximal del piramidal y/o un solapamiento LP (fig. 1). Raramente existe un gap entre el semilunar y el

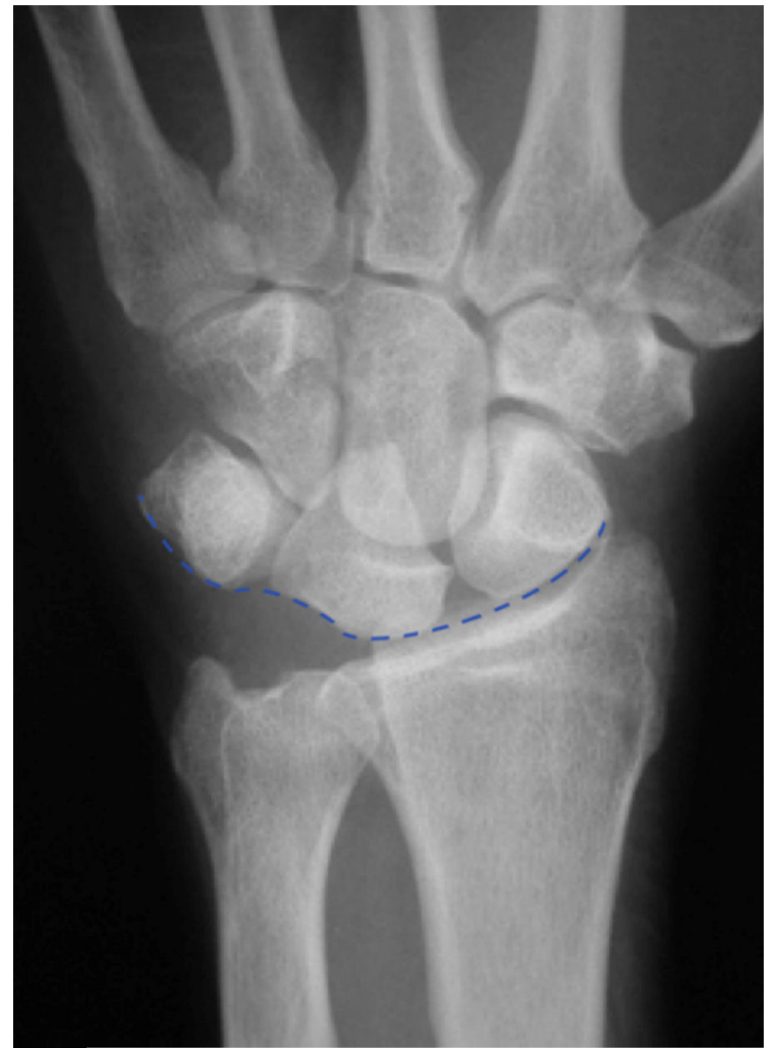

Figura 1 Imagen de una disociación lunopiramidal en la que el arco de Gilula formado por la superficie proximal de la hilera proximal del carpo se ve alterado.

piramidal. El semilunar adquiere forma de semiluna por la posición en flexión del semilunar ${ }^{7}$ (fig. 2).

En las radiografías dinámicas puede observarse un aumento de la traslación proximal del piramidal en la desviación ulnar, mientras que en la desviación radial se observa un aumento de la flexión palmar del escafoides y el semilunar, a la vez que el piramidal está normalmente alineado.

En las proyecciones laterales, si existe una disociación LP el semilunar adopta una posición en flexión y el ángulo LP, que en condiciones normales es de $14^{\circ}$, adquiere valores negativos $^{8}$ (figs. 3 y 4 ).

\section{Artrografía}

Puede mostrar un paso del contraste entre la articulación radiocarpiana y mediocarpiana, pero esto no es específico ya que puede indicar desde una rotura aguda hasta una rotura crónica relacionada con la edad o con un síndrome de impactación ulnocarpiano ${ }^{12}$.

\section{Videofluoroscopia}

En lesiones sin deformidad estática se puede observar un «clunc» en los últimos grados de desviación ulnar máxima de la muñeca con un repentino cambio de posición del semilunar de flexión a extensión.

\section{Gammagrafía ósea}

Puede aparecer un aumento de captación en el intervalo LP. Es útil cuando el resto de los exámenes radiológicos son negativos. 

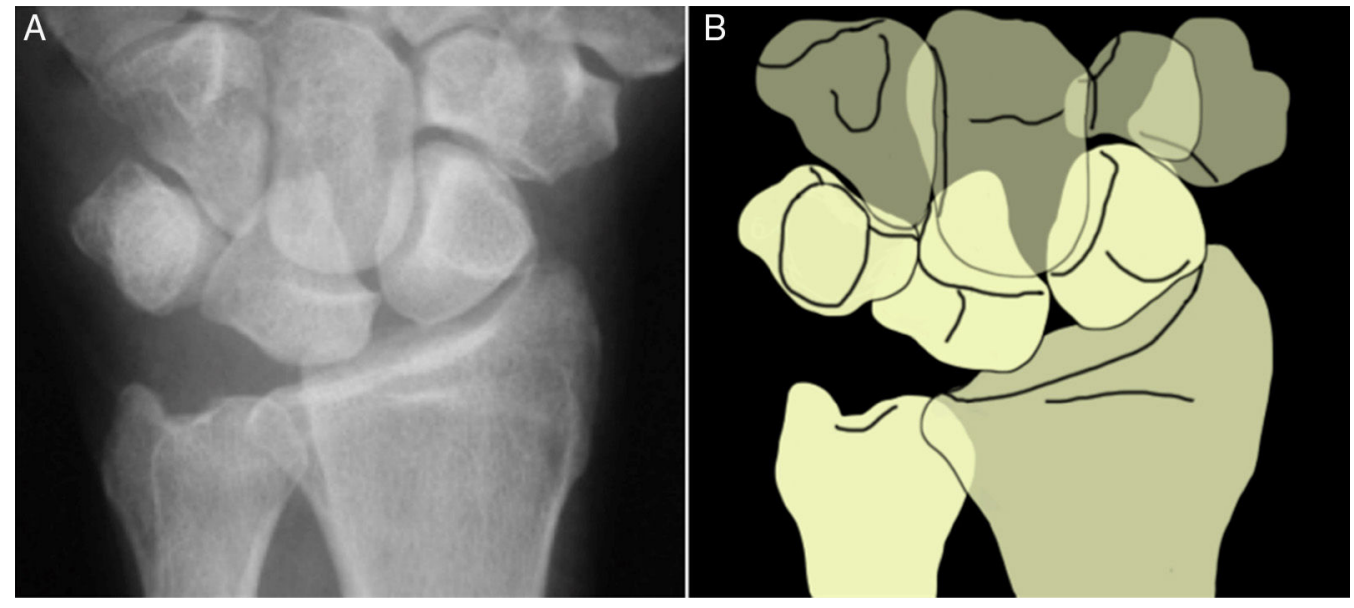

Figura 2 Semilunar con forma de «semiluna» en la que la parte más triangular de la figura constituye el borde dorsal del semilunar que está superpuesto a la porción distal y dorsal del hueso grande.

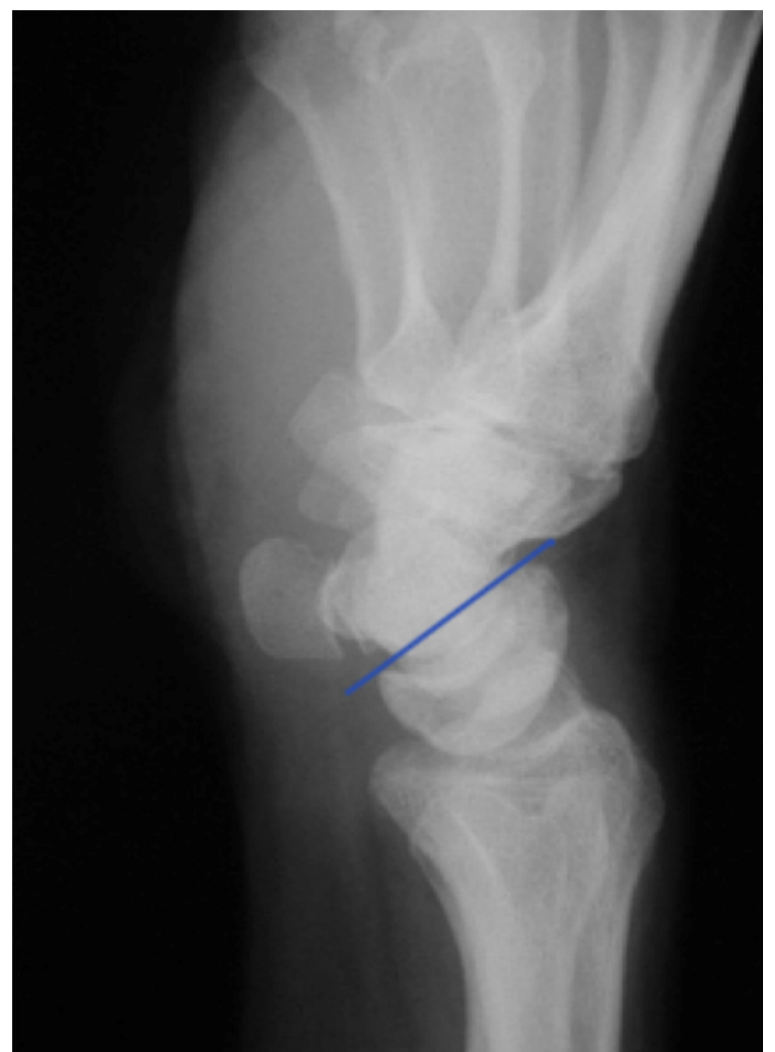

Figura 3 Radiografía lateral en la que se observa una deformidad estática en VISI con el semilunar en flexión.

\section{Resonancia magnética}

Se puede identificar una rotura cuando el segmento ligamentoso está ausente o existe una discontinuidad ocupada por líquido que aparece como una hiperintensidad de la señal en secuencias potenciadas en T2. También una distorsión de su anatomía o del curso del ligamento pueden ser indicativos de rotura. Con los estudios obtenidos con RMN de 3 teslas se obtiene una especificidad de hasta el 100\% comparado con la artroscopia de muñeca y una sensibilidad del 22 al $82 \%$. Si se incluye la artroRMN en los estudios, esta sensibilidad aumenta del 50 al 100\%, siendo mayor en las roturas completas que en las parciales ${ }^{13}$. Por otra parte, la obtención de imágenes en un plano oblicuo axial (perpendicular al intervalo EL o LP) en vez de un plano axial (perpendicular al radio) aumenta la seguridad en la detección de lesiones de los ligamentos interóseos ${ }^{14}$.

\section{Diagnóstico artroscópico}

La artroscopia de muñeca constituye el gold standard para el diagnóstico de las lesiones de los ligamentos interóseos. El ligamento LP es visualizado en la articulación mediocarpiana a través del portal dorsal mediocarpiano radial, mientras que en la radiocarpiana se puede emplear el portal $6 \mathrm{R}$ o el 4/5 desviando la óptica hacia proximal y ulnar. Con esta visión se observa fácilmente la porción membranosa e incluso la porción dorsal del ligamento LP. Esta visión también aporta información sobre el estado de la superficie articular proximal del piramidal (fig. 5).

Se suele utilizar la clasificación de Geissler (tabla 1) para determinar el grado de inestabilidad. Sin embargo, la valoración del grado de separación LP tiene 2 inconvenientes que lo hacen mucho menos «fiable» que el grado de separación EL. El primero es que el gancho palpador no entra a través del portal mediocarpiano ulnar de forma paralela al intervalo LP como lo hace en el intervalo EL, sino que entra de forma oblicua, lo que provoca que la superficie proximal del piramidal le impida en cierto modo introducirlo en el intervalo LP y girarlo (vídeo1). El segundo inconveniente radica en las distintas morfologías que presenta el semilunar. Existen 2 tipos de semilunar, según la descripción de Viegas et al. ${ }^{15}$ : un tipo ।, sin carilla articular para el ganchoso, y un tipo II, con una carilla articular para el hueso grande y otro para el ganchoso. En el caso de existir un semilunar tipo ॥ con la carilla para el ganchoso cerca de la articulación LP, puede crear una falsa sensación de separación (fig. 6). Por eso, es fundamental realizar siempre una visión cenital, saltando la cresta de la carilla articular del ganchoso (vídeo 2).

La artroscopia de muñeca también permite ver el estado de los estabilizadores secundarios, esto es, el ligamento radiocarpiano dorsal y el intercarpiano dorsal a través de los 


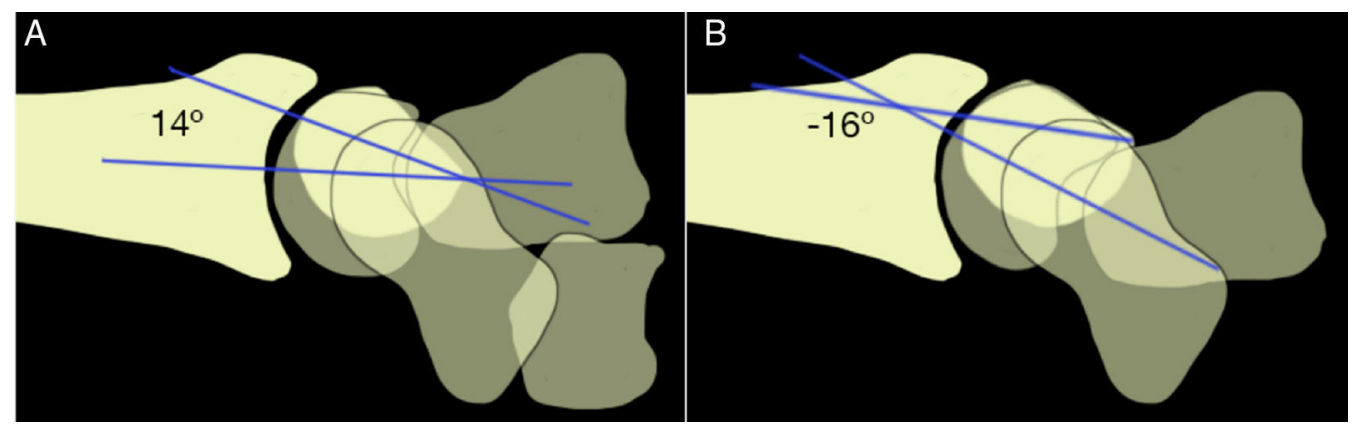

Figura 4 A) En una muñeca normal el ángulo lunopiramidal medio es de 14. B) En la disociación lunopiramidal el ángulo lunopiramidal adquiere valores negativos, con una media de $-16^{\circ 8}$.
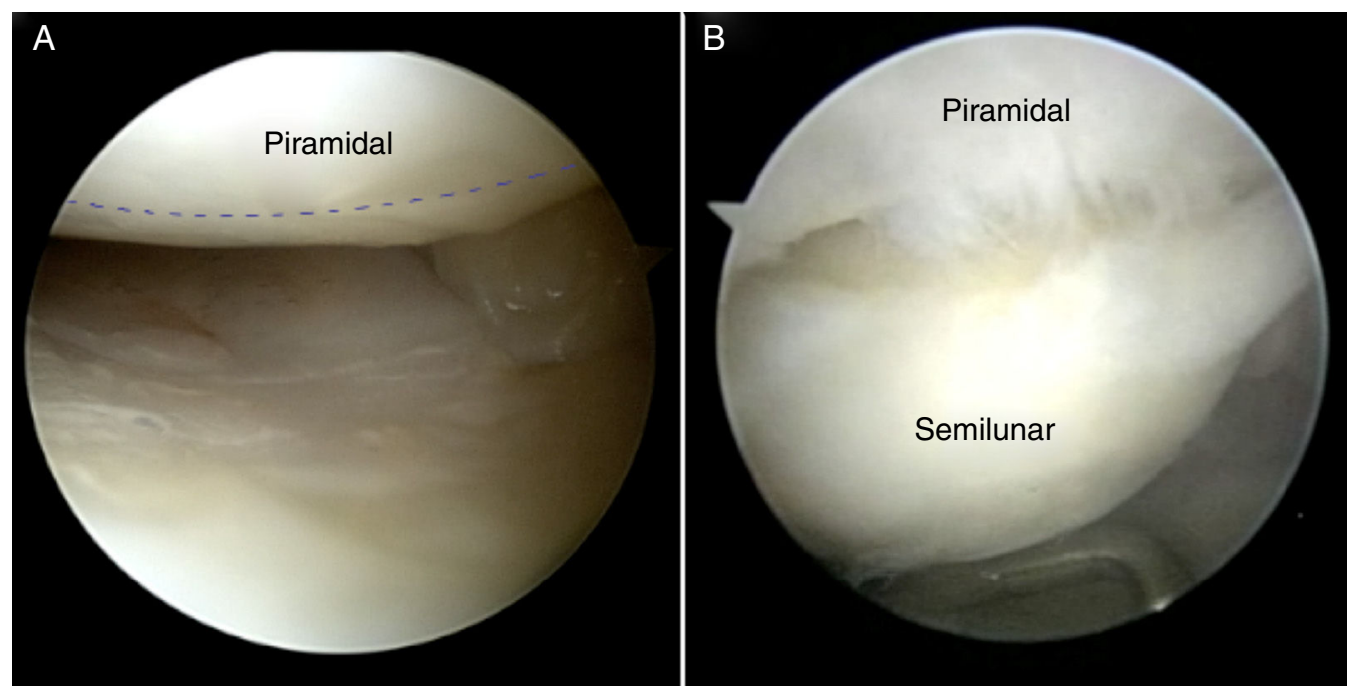

Figura 5 Visión desde el portal 6R en muñeca derecha. A) Porción membranosa del ligamento LP normal marcado por la línea discontinua en azul. B) Rotura del ligamento LP.

portales volares. Los autores de este trabajo recomiendan la utilización del portal volar central para este fin $^{16}$ (fig. 7). Además de realizar una valoración estática de la situación del ligamento LP, se puede hacer una valoración dinámica que en muchas ocasiones aporta un valor adicional y/o el diagnóstico definitivo. El Ballotment test artroscópico del ligamento LP (vídeo 3) tiene aún más valor que el del EL, porque como se ha comentado previamente, la valoración del gap es mucho menos fiable.

Se realiza en la articulación mediocarpiana, el artroscopio se coloca sobre el semilunar, entrando a través del portal dorsal mediocarpiano radial. Se retira la tracción y se realiza un movimiento anteroposterior del LP, fijando el semilunar. Si no existe una inestabilidad importante, los 2 huesos se moverán a la par, pero si existe inestabilidad el piramidal se moverá, quedando el semilunar fijo.

Por último, la artroscopia de muñeca permite diagnosticar lesiones combinadas de los ligamentos LP y EL, lo que no constituye una situación excepcional. Esta lesión combinada ha sido descrita por Badia y Khanchandani ${ }^{17}$ como «semilunar flotante» porque las inserciones ligamentosas en ambos lados de la semilunar están ausentes y el semilunar «flota» en una posición neutra. Para el diagnóstico de un semilunar «flotante» y desinsertado del escafoides y

Tabla 1 Clasificación de Geissler de las lesiones de los ligamentos interóseos ${ }^{43}$

\begin{tabular}{ll}
\hline Grado de lesión & Visión artroscópica \\
\hline Lesión grado I & $\begin{array}{l}\text { Atenuación o hemorragia del ligamento escafolunar y pérdida de su concavidad en la articulación } \\
\text { radiocarpiana. Sin incongruencia mediocarpiana }\end{array}$ \\
Lesión grado II & $\begin{array}{l}\text { Incongruencia mediocarpiana inferior a la anchura de un gancho palpador } \\
\text { Lesión grado III }\end{array}$ \\
$\begin{array}{l}\text { Separación o incongruencia entre los huesos del carpo, tanto en el espacio radiocarpiano como en el } \\
\text { medioniano. El gancho palpador puede pasar entre los huesos, pero no la óptica del artroscopio }\end{array}$ \\
Lesión grado Iv & La separación entre los huesos del carpo es mayor: incluso puede pasar un artroscopio de 2,7 mm \\
\hline
\end{tabular}



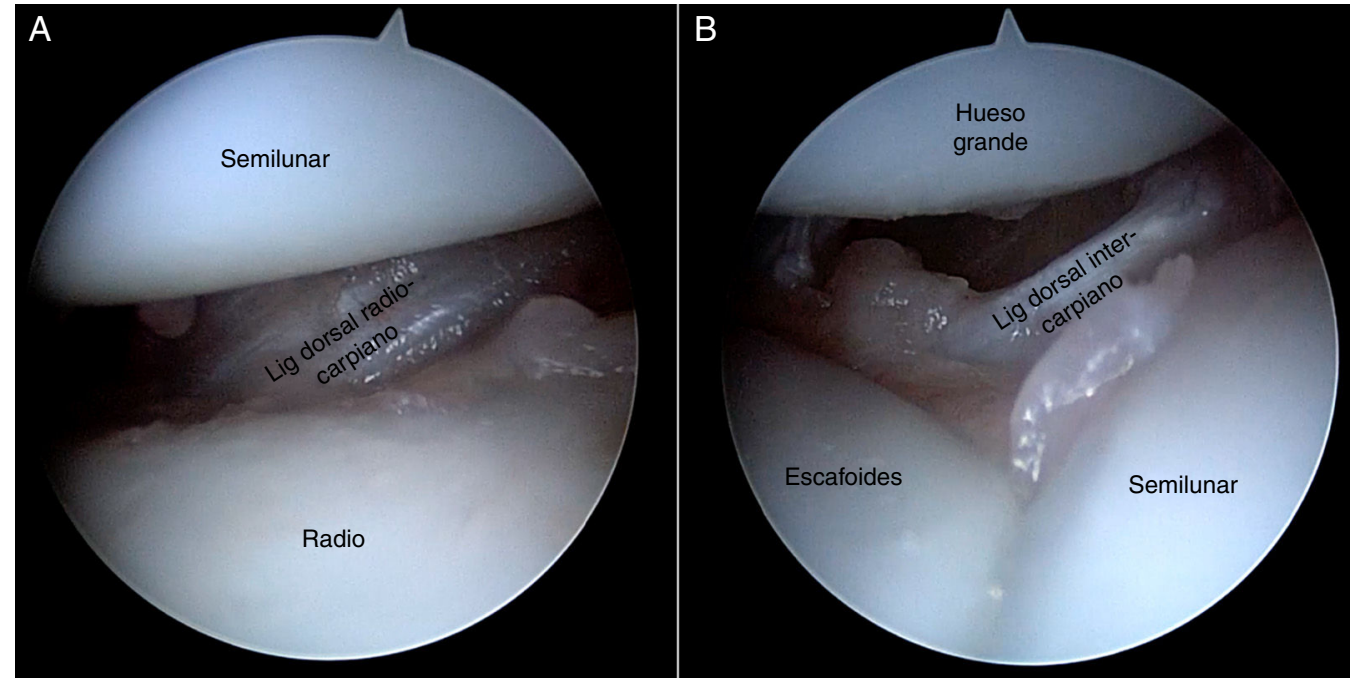

Figura 6 Visión de los ligamentos dorsales desde el portal volar central en muñeca derecha. A) Visión del ligamento radiocarpiano dorsal en la articulación radiocarpiana. B) Visión del ligamento intercarpiano dorsal en la articulación mediocarpiana.

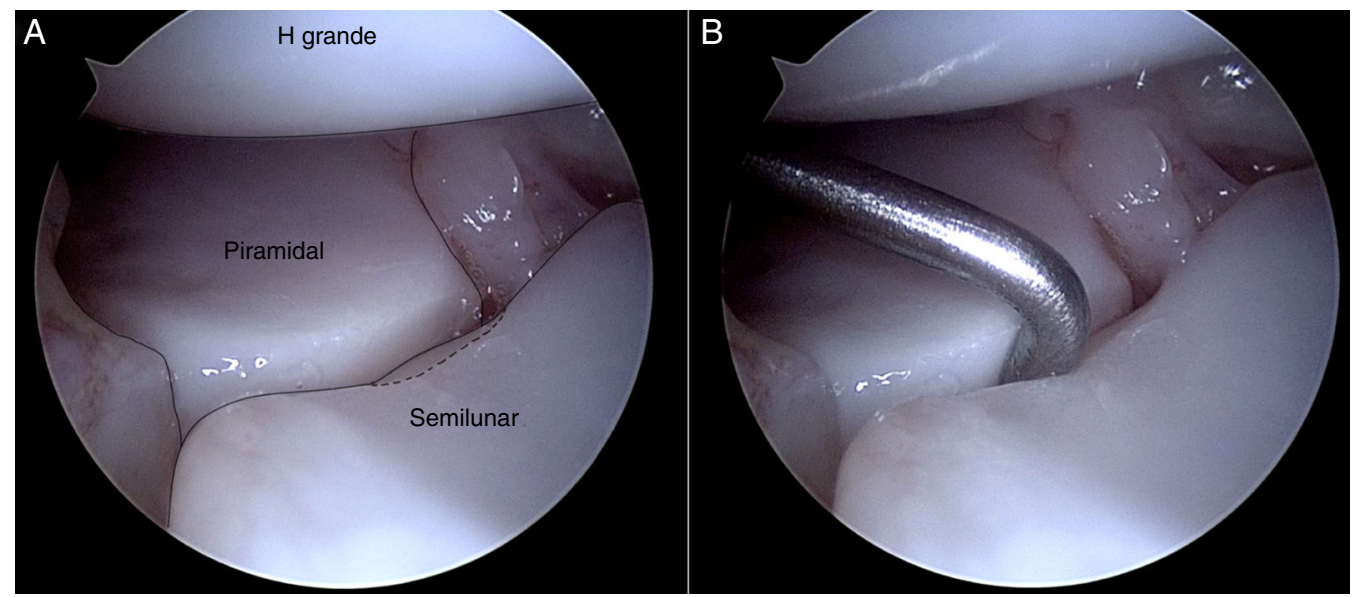

Figura 7 Visión desde el portal DMR en muñeca izquierda. A) Carilla en semilunar para el ganchoso marcada como línea discontinua. B) Al introducir el gancho palpador puede dar la falsa sensación de que este se introduce más de lo normal, pero es debido a la presencia de una carilla para el ganchoso muy pronunciada.

piramidal puede ser útil el «signo de la mecedora» ${ }^{18}$ (vídeo 4). El artroscopio se coloca sobre el piramidal, pero sin apoyarse sobre él. Se debe obtener una visión de la articulación EL, del hueso semilunar y de la articulación LP. El gancho explorador entra a través del portal mediocarpiano radial. En este momento la tracción de la torre se libera y se realiza, con el palpador, un movimiento de oscilación repetido sobre el semilunar de posterior a anterior y de anterior a posterior. En una muñeca normal, el semilunar permanece estático cuando se realiza este movimiento oscilante. Por el contrario, si hay un «semilunar flotante», el semilunar se moverá de forma similar a un «mecedora», mientras que el escafoides y el piramidal permanecen inmóviles.

\section{Tratamiento}

No existe un consenso en cuanto a qué tratamiento es la mejor opción en los casos de lesión del ligamento LP, ya que los estudios disponibles son de diseño retrospectivo y sin medidas de evolución estandarizadas. Entre las opciones de tratamiento se encuentran la inmovilización, el desbridamiento artroscópico aislado o asociado a otros procedimientos, la reparación o reconstrucción del ligamento, las artrodesis parciales y la osteotomía de acortamiento ulnar.

\section{Tratamiento conservador}

El manejo inicial de la mayoría de las lesiones agudas y crónicas del ligamento LP sin inestabilidad asociada puede ser conservador, con un período de inmovilización inicial con yeso $u$ ortesis que ejerza presión sobre el pisiforme para mantener el alineamiento de la hilera proximal del carpo. Reagan et al. ${ }^{8}$ consiguen buenos resultados en 6 de 7 pacientes con lesiones agudas y en uno de 4 pacientes con 
lesiones crónicas del ligamento LP tratadas con inmovilización durante 6 semanas.

En los últimos años se está implicando al extensor ulnar del carpo como estabilizador dinámico de la articulación LP, cuya potenciación sería beneficiosa en las inestabilidades dinámicas del ligamento LP. Este mecanismo se explica porque en situaciones de carga axial o contracción de los músculos motores de la muñeca con el ligamento LP lesionado, el piramidal se flexiona y se supina guiado por la traslación palmar del ganchoso durante la supinación de la hilera distal del carpo. La contracción del extensor ulnar del carpo produce una pronación de la hilera distal del carpo, lo que provoca una extensión del piramidal y estabiliza la articulación LP ${ }^{19,20}$.

En lesiones agudas o crónicas con inestabilidad asociada o lesiones crónicas en las que persista la sintomatología a pesar del tratamiento conservador está indicado el tratamiento quirúrgico.

\section{Artroscopia}

Las lesiones agudas grado III y ıv de Geissler se han manejado de forma favorable con reducción artroscópica y estabilización con agujas de Kirschner durante 8 semanas $^{21}$. El problema fundamental es que este tipo de lesiones, si se dan de forma aislada, se suelen diagnosticar de forma tardía. Se han descrito buenos resultados para el tratamiento artroscópico de las lesiones crónicas parciales del ligamento LP. Estos resultados son más impredecibles en las lesiones crónicas completas. Entre los procedimientos artroscópicos descritos están el desbridamiento aislado o asociado a reducción y estabilización con agujas, o a la termocoagulación del ligamento LP.

\section{Desbridamiento aislado}

Weiss et al. ${ }^{22}$ comunican los resultados del desbridamiento artroscópico de roturas parciales y completas de los ligamentos EL y LP. Encuentran una mejoría clínica o una resolución completa de los síntomas en 7 de 9 (78\%) pacientes con lesión completa del ligamento LP y en los 6 pacientes $(100 \%)$ con lesiones parciales del mismo en un periodo de seguimiento medio de 27 meses.

Ruch y Poehling ${ }^{23}$ encuentran una mejoría completa de los síntomas en 11 pacientes de 14 con lesiones parciales crónicas de los ligamentos EL o LP después del desbridamiento artroscópico en un periodo de seguimiento medio de 34 meses.

A diferencia de los buenos resultados obtenidos en los estudios anteriores, Westkaemper et al. ${ }^{24}$ obtienen malos resultados con el desbridamiento artroscópico de las lesiones completas del ligamento LP en 4 de 5 casos en un periodo de seguimiento medio de 15,4 meses.

Desbridamiento más estabilización con agujas Osterman y Seidman ${ }^{25}$ llevan a cabo un desbridamiento del ligamento LP más estabilización con agujas de Kirschner en 20 pacientes con rotura completa del ligamento LP sin deformidad en VISI asociada. Encuentran un $80 \%$ de buenos resultados en un periodo de seguimiento medio de 2 años.
Desbridamiento más termocoagulación

Lee et $a .^{26}$ realizan un desbridamiento artroscópico más termocoagulación en roturas parciales de los ligamentos LP y EL en 16 casos. Todos los pacientes experimentan una mejoría de la clínica y una resolución completa de los síntomas en reposo en un periodo medio de seguimiento de 52,8 meses.

\section{Reparación del ligamento lunopiramidal}

Está indicada en lesiones agudas completas con un ligamento reparable, asociado o no a una capsulodesis. Si la porción volar, que es la más robusta, se encuentra también lesionada, en ocasiones una doble vía de abordaje puede ser de utilidad. A pesar de estas indicaciones, la reparación del ligamento LP se ha descrito en casos de roturas crónicas y asociada a un alto porcentaje de complicaciones ${ }^{27}$.

Reagan et al. 8 llevan a cabo una reparación del ligamento por vía dorsal en 7 casos de lesiones crónicas, refrescando la inserción ligamentosa en el piramidal y coaptando el ligamento mediante suturas transóseas y refuerzo de la sutura con agujas de Kirschner. Obtienen una mejoría del dolor en 6 de 7 casos en un periodo de seguimiento entre 18 y 123 meses. A pesar de ello reconocen que es una técnica más bien difícil, con la inseguridad en cuanto a la fuerza tensil aplicada y su duración, por lo que la recomiendan para lesiones parciales en pacientes sin gran demanda funcional.

Shin et al. ${ }^{27}$ realizan una reparación del ligamento LP en 27 casos de forma similar a la descrita por Reagan et al. ${ }^{8}$. De estos, solo uno es reparado en la fase aguda. Encuentran un $40,7 \%$ de complicaciones que incluyen 4 fallos $(14,8 \%)$ de la reparación después de un traumatismo adicional en un periodo medio de seguimiento de 6,7 años.

\section{Capsulodesis}

Puede realizarse como procedimiento adyuvante a una reparación, o de forma aislada en casos de roturas crónicas no reparables con lesión de los estabilizadores secundarios (ligamento radiocarpiano dorsal e intercarpiano dorsal). Los procedimientos abiertos descritos intentan reforzar la porción dorsal del ligamento LP, mientras que el artroscópico refuerza la porción volar.

Moskal et al. ${ }^{28}$ realizan una capsulodesis volar mediante una plicatura artroscópica de los ligamentos ulnosemilunar, ulnopiramidal y ulnohuesogrande asociada a una reducción y estabilización de la articulación LP con agujas en 20 casos con roturas parciales del ligamento LP. Obtienen resultados buenos o excelentes en 18 casos en un periodo de seguimiento medio de 3,1 años.

De Smet et al. ${ }^{29}$ comunican los resultados de 13 capsulodesis dorsales en lesiones crónicas del ligamento LP, según la técnica descrita por Sennwald y Fisher ${ }^{11}$ en la que se obtiene un flap de la porción más distal del retináculo extensor con base radial, que se ancla al piramidal y al semilunar mediante anclajes óseos. Se realiza una reducción más estabilización con agujas añadida en 5 capsulodesis. La satisfacción del paciente es pobre en 5, moderada en 5 y excelente en 3 casos. Tres pacientes necesitan un 
procedimiento secundario en un período de seguimiento medio de 3,2 años.

Antti-Poika et al. ${ }^{30}$ realizan un capsulodesis dorsal de forma similar a la descrita por Sennwald y Fisher ${ }^{31}$ para roturas crónicas del ligamento LP grados। a III de Geissler en 26 pacientes. Los resultados son buenos o excelentes en 23 pacientes en un período de seguimiento medio de 39 meses.

Omokawa et al. ${ }^{32}$ diseñan una capsulodesis dorsal con el avance del ligamento radiocarpiano dorsal, que se fija al semilunar y al piramidal mediante anclajes óseos después de reducir y estabilizar la articulación LP con agujas de Kirschner. Describen los resultados de este procedimiento en 11 pacientes con inestabilidad crónica predinámica, siendo buenos o excelentes en 7 casos en un período de seguimiento medio de 31 meses.

\section{Reconstrucción del ligamento lunopiramidal}

La reconstrucción del ligamento LP es una alternativa a la artrodesis en el caso de lesiones completas no reparables sin cambios degenerativos asociados, con la ventaja de preservar la movilidad LP.

Reagan et al. ${ }^{8}$ realizan una reconstrucción del ligamento LP con un injerto tendinoso de base distal del flexor ulnar del carpo que atraviesa de forma vertical el ganchoso y el piramidal. Lo realizan en 3 roturas crónicas, 2 de ellas con disociación LP asociada (rotura de los constrictores ligamentosos entre el piramidal y el semilunar con pérdida de la relación LP normal en la radiografía simple). Obtienen buenos resultados en los 3 casos con un seguimiento de 22 a 54 meses. En los casos con disociación asociada, persiste el malalineamiento del carpo en los controles radiográficos posteriores.

Shin et al. ${ }^{27}$ describen una reconstrucción con un injerto tendinoso de base distal procedente del extensor ulnar del carpo, colocado a través de túneles en el piramidal y el semilunar en 8 casos con roturas crónicas del ligamento LP. Obtienen mejores resultados que con la artrodesis LP o la reparación del ligamento LP en un periodo medio de seguimiento de 6,7 años.

Shahane et al. ${ }^{33}$ realizan una plastia con un injerto de base distal del extensor ulnar del carpo que atraviesa el piramidal y la porción dorsal del complejo del fibrocartílago triangular. Su propósito no es recrear la anatomía del ligamento LP sino mejorar la cinemática permitiendo una movilidad más anatómica entre la primera hilera del carpo. Comunican los resultados de este procedimiento en 46 pacientes con inestabilidad LP dinámica. Obtienen resultados buenos o excelentes en $29(63 \%)$ y satisfactorios en 11 (24\%) en un periodo medio de seguimiento de 39,1 meses. Cinco pacientes desarrollan problemas en la articulación pisopiramidal que los autores atribuyen a una excesiva tensión de la plastia.

Pilný et al. ${ }^{34}$ describen una modificación de la plastia con el extensor ulnar del carpo descrita por Shin et al. ${ }^{27}$ en la que la misma se ancla al semilunar mediante anclajes óseos sin la realización de un túnel. Llevan a cabo el procedimiento en 19 casos con inestabilidad crónica. No encuentran malos resultados en ningún caso, aunque su seguimiento es de 4 meses.

\section{Artrodesis lunopiramidal}

Reservada para pacientes con inestabilidad crónica y dolor persistente en los que han fallado otros procedimientos, o pacientes que han desarrollado cambios degenerativos incipientes.

La artrodesis LP está fundamentada en el hecho de que los pacientes con coalición LP congénita están habitualmente asintomáticos, y que bajo circunstancias normales existe escaso movimiento entre el semilunar y el piramidal ${ }^{7}$.

El material utilizado para la artrodesis varía desde agujas de Kirschner ${ }^{8,11,35}$ a tornillos de compresión ${ }^{31,36}$ o grapas gr,37. $^{29}$. Se aconseja suplementar la fusión con injerto óseo para disminuir el riesgo de seudoartrosis ${ }^{31}$.

Debido a que el semilunar y el piramidal se mueven de forma sincrónica, la artrodesis LP produce una pérdida de la movilidad poco significativa, en torno al $80 \%$ respecto al lado contralateral ${ }^{35,38}$. A pesar de ello, se postula que el ligamento LP amortigua las fuerzas del lado ulnar de la muñeca. Taleisnik teoriza que la fusión LP produce una barra rígida que puede aumentar la presión sobre el complejo fribrocartílago triangular y la cabeza de la ulna, y conducir a un síndrome de impactación secundario ${ }^{11}$. A este respecto, Favero et al. comunican la aparición de un síndrome de impactación ulnocarpiana que requiere cirugía posterior en el $14 \%$ de los casos, y Shin et al. ${ }^{27}$ en el $22 \%$.

A pesar de ser técnicamente menos demandante que otros procedimientos, no está exenta de complicaciones, que incluyen una cifra de seudoartrosis de hasta el $57 \% 27,29,31,37-40$.

Además de la alta tasa de complicaciones que presentan algunos trabajos, la evolución comunicada no es uniformemente favorable. Mientras que la evolución es favorable en algunas series, con resultados satisfactorios en más del $70 \%$ de los $\operatorname{casos}^{35,36,38}$, otros autores comunican resultados desfavorables, desaconsejando esta técnica frente a otros procedimientos $^{27,29,31}$.

\section{Osteotomía de acortamiento ulnar}

Este procedimiento en el contexto de una lesión del ligamento LP está basado en el estudio biomecánico de Gupta et al. ${ }^{41}$, que sugiere que la osteotomía de acortamiento ulnar reduce la movilidad relativa del semilunar y piramidal al aumentar la tensión en los ligamentos ulnosemilunar y ulnopiramidal.

Mirza et al. ${ }^{42}$ llevan a cabo una osteotomía de acortamiento ulnar en 53 pacientes con lesión crónica del ligamento LP sin cambios degenerativos asociados. Obtienen resultados buenos o excelentes en el $83 \%$ de los casos en un periodo de seguimiento medio de 36 meses.

Aunque es difícil establecer un algoritmo de tratamiento basado en los estudios disponibles debido a su falta de homogeneidad en el diseño, podemos realizar una recomendación de un procedimiento u otro basándonos en la cronicidad de la lesión y en el tipo de rotura del ligamento LP (tabla 2), aunque son necesarios estudios a largo plazo prospectivos aleatorizados con medidas de evolución validadas que establezcan cuáles son los procedimientos más idóneos para cada caso. 
Tabla 2 Algoritmo de tratamiento basado en el tipo de lesión y la cronicidad

\begin{tabular}{ll} 
Tipo de lesión & Tratamiento \\
\hline Aguda completa & Reparación primaria \pm capsulodesis \\
Crónica parcial & Reducción artroscópica + agujas \\
& Inmovilización \\
& Desbridamiento artroscópico/termocoagulación/reducción y \\
& estabilización con agujas \\
Crónica completa sin deformidad en VISI estática & Capsulodesis \\
& Reconstrucción del ligamento LP \\
& Artrodesis LP \\
Crónica completa con deformidad en VISI estática & Osteotomía de acortamiento ulnar \\
& Artrodesis 4 esquinas \\
& Resección hilera proximal del carpo
\end{tabular}

\section{Responsabilidades éticas}

Protección de personas y animales. Los autores declaran que para esta investigación no se han realizado experimentos en seres humanos ni en animales.

Confidencialidad de los datos. Los autores declaran que en este artículo no aparecen datos de pacientes.

Derecho a la privacidad y consentimiento informado. Los autores declaran que en este artículo no aparecen datos de pacientes.

\section{Conflicto de intereses}

Los autores declaran que no existe conflicto de intereses en la elaboración de este trabajo.

\section{Anexo. Material adicional}

Se puede consultar material adicional a este artículo en su versión electrónica disponible en doi:10.1016/j.ricma. 2016.09.003.

\section{Bibliografía}

1. Ritt MJ, Bishop AT, Berger RA, Linscheid RL, Berglund LJ, An KN. Lunotriquetral ligament properties: A comparison of three anatomic subregions. J Hand Surg Am. 1998;23:425-31.

2. Horii E, Garcia-Elias M, An KN, Bishop AT, Cooney WP, Linscheid RL, et al. A kinematic study of luno-triquetral dissociations. J Hand Surg Am. 1991;16:355-62.

3. Viegas SF, Patterson RM, Peterson PD, Pogue DJ, Jenkins DK, Sweo TD, et al. Ulnar-sided perilunate instability: An anatomic and biomechanic study. J Hand Surg Am. 1990;15:268-78.

4. Trumble TE, Bour CJ, Smith RJ, Glisson RR. Kinematics of the ulnar carpus related to the volar intercalated segment instability pattern. J Hand Surg Am. 1990;15:384-92.

5. Nicoson M, Moran S. Diagnosis and treatment of acute lunotriquetral ligament injuries. Hand Clin. 2015;31:467-76.

6. Mayfield JK, Johnson RP, Kilcoyne RK. Carpal dislocations: Pathomechanics and progressive perilunar instability. J Hand Surg Am. 1980;5:226-41.
7. Shin AY, Battaglia MJ, Bishop AT. Lunotriquetral instability: Diagnosis and treatment. J Am Acad Orthop Surg. 2000;8:170-9.

8. Reagan DS, Linscheid RL, Dobyns JH. Lunotriquetral sprains. J Hand Surg Am. 1984;9:502-14.

9. Weiss LE, Taras JS, Sweet SOL. Lunotriquetral injuries in the athlete. Hand Clin. 1997; 16:433-8.

10. Christodoulou L, Bainbridge LC. Clinical diagnosis of triquetrolunate ligament injuries. J Hand Surg Br. 1999;24:598-600.

11. Ambrose L, Posner M. Lunate-triquetral and midcarpal joint instability. Hand Clin. 1992;8:653-68.

12. García-Elías M. Carpal instability. En: Wolfe S, Hotchkiss R, Pederson W, Kozin S, editores. Green's Operative Hand Surgey. 6th ed New York, Churchill Livingstone: Elsevier; 2011. p. 465-522.

13. Ringler MD, Murthy NS. MR imaging of wrist ligaments. Magn Reson Imaging Clin N Am. 2015;23:367-91.

14. Lee RKL, Griffith JF, Ng AWH, Law EKC, Tse WL, Wong CWY, et al. Intrinsic carpal ligaments on MR and multidetector CT arthrography: Comparison of axial and axial oblique planes. Eur Radiol. 2016 [epub ahead of print].

15. Viegas SF, Wagner K, Patterson R, Peterson P. Medial (hamate) facet of the lunate. J Hand Surg Am. 1990;15:564-71.

16. Corella F, Ocampos M, del Cerro M, Larrainzar-Garijo R, Vázquez T. Volar central portal in wrist arthroscopy. J Wrist Surg. 2016;5:80-90.

17. Badia A, Khanchandani P. The floating lunate: Arthroscopic treatment of simultaneous complete tears of the scapholunate and lunotriquetral ligaments. Hand. 2009;4:250-5.

18. Corella F, del Cerro M, Ocampos M, Larrainzar-Garijo R. The rocking chair sign for floating lunate. J Hand Surg Am. 2015;40:2318-9.

19. León-López M, Salvá-Coll G, García-Elías M, Lluch-Bergaradá A, Llusá-Pérez M. Role of the extensor carpi ulnaris in the stabilization of the lunotriquetral joint. An experimental study. J Hand Ther. 2013;26:312-7.

20. Espluglas M, García-Elías M, Lluch A, Llusá-Pérez M. Role of muscles in the stabilization of ligament-deficient wrists. J Hand Ther. 2016;29:166-74.

21. Slutsky DJ, Trevare J. Scapholunate and lunotriquetral injuries: Arthroscopic and open management. Sports Med Arthrosc. 2014;22:12-21.

22. Weiss AP, Sachar K, Glowacki KA. Arthroscopic debridement alone for intercarpal ligament tears. J Hand Surg Am. 1997;22:344-9.

23. Ruch D, Poehling G. Arthroscopic management of partial scapholunate and lunotriquetral injuries of the wrist. J Hand Surg Am. 2008;90:412-7.

24. Westkaemper JG, Mitsionis G, Giannakopoulos PN, Sotereanos DG. Wrist arthroscopy for the treatment of ligament 
and triangular fibrocartilage complex injuries. Arthroscopy. 1998;14:479-83.

25. Osterman A, Seidman G. The role of arthroscopy in the treatment of lunatotriquetral ligament injuries. Hand Clin. 1995;11:41-50.

26. Lee JI, Nha KW, Lee GY, Kim BH, Kim JW, Park JW. Long-term outcomes of arthroscopic debridement and thermal shrinkage for isolated partial intercarpal ligament tears. Orthopedics. 2012;35:1204-9.

27. Shin AY, Weinstein LP, Berger RA, Bishop AT. Treatment of isolated injuries of the lunotriquetral ligament. A comparison of arthrodesis, ligament reconstruction and ligament repair. $J$ Bone Jt Surg Br. 2001;83:1023-8.

28. Moskal MJ, Savoie FH, Field LD. Arthroscopic capsulodesis of the lunotriquetral joint. Clin Sports Med. 2001;20:141-53.

29. De Smet L, Janssens I, van de Sande W. Chronic lunotriquetral ligament injuries: Arthrodesis or capsulodesis. Acta Chir Belg. 2005;105:79-81.

30. Antti-Poika I, Hyrkas J, Virkki L, Ogino D, Konttinen Y. Correction of chronic lumotriquetral instability using extensor retinacular split: A retrospective study of 26 patients. Acta Orthop Belg. 2007;73:451-7.

31. Sennwald GR, Fischer M, Mondi P. Lunotriquetral arthrodesis. A controversial procedure. J Hand Surg Br. 1995;20:755-60.

32. Omokawa S, Fujitani R, Inada Y. Dorsal radiocarpal ligament capsulodesis for chronic dynamic lunotriquetral instability. J Hand Surg Am. 2009;34:237-43.

33. Shahane SA, Trail IA, Takwale VJ, Stilwell JH, Stanley JK. Tenodesis of the extensor carpi ulnaris for chronic, post-traumatic lunotriquetral instability. J Bone Joint Surg Br. 2005;87:1512-5.
34. Pilný J, Svarc A, Perina M, Siller J, Visna P. Chronic lunotriquetral instability of the wrist. Presentation of our method of treatment. Acta Chir Orthop Traumatol Cech. 2009;76: 208-11.

35. Guidera PM, Watson HK, Dwyer TA, Orlando G, Zeppieri J, Yasuda M. Lunotriquetral arthrodesis using cancellous bone graft. J Hand Surg Am. 2001;26:422-7.

36. Pin P, Young V, Gilula L, Weeks P. Management of chronic lunotriquetral ligament tears. J Hand Surg Am. 1989;14:77-83.

37. Vandesande W, De Smet L, van Ransbeeck H. Lunotriquetral arthrodesis, a procedure with a high failure rate. Acta Orthop Belg. 2001;67:361-7.

38. Kirschenbaum D, Coyle M, Leddy J. Chronic lunotriquetral instability: Diagnosis and treatment. J Hand Surg Am. 1993;18:1107-12.

39. Nelson D, Manske P, Pruitt D, Gilula L, Martin R. Lunotriquetral arthrodesis. J Hand Surg Am. 1993;18:1113-20.

40. McAuliffe J, Dell P, Jaffe R. Complications of intercarpal arthrodesis. J Hand Surg Am. 1993;18:1121-8.

41. Gupta R, Bingenheimer E, Fornalski S, Mc Garry M, Osterman A, Lee $T$. The effect of ulnar shortening on lunate and triquetrum motion-a cadaveric study. Clin Biomech. 2005;20:839-45.

42. Mirza A, Mirza JB, Shin AY, Lorenzana DJ, Lee BK, Izzo B. Isolated lunotriquetral ligament tears treated with ulnar shortening osteotomy. J Hand Surg Am. 2013;38:1492-7.

43. Geissler WB, Freeland AE, Savoie FH, McIntyre LW, Whipple TL. Intracarpal soft-tissue lesions associated with an intra-articular fracture of the distal end of the radius. J Bone Joint Surg Am. 1996;78:357-65. 